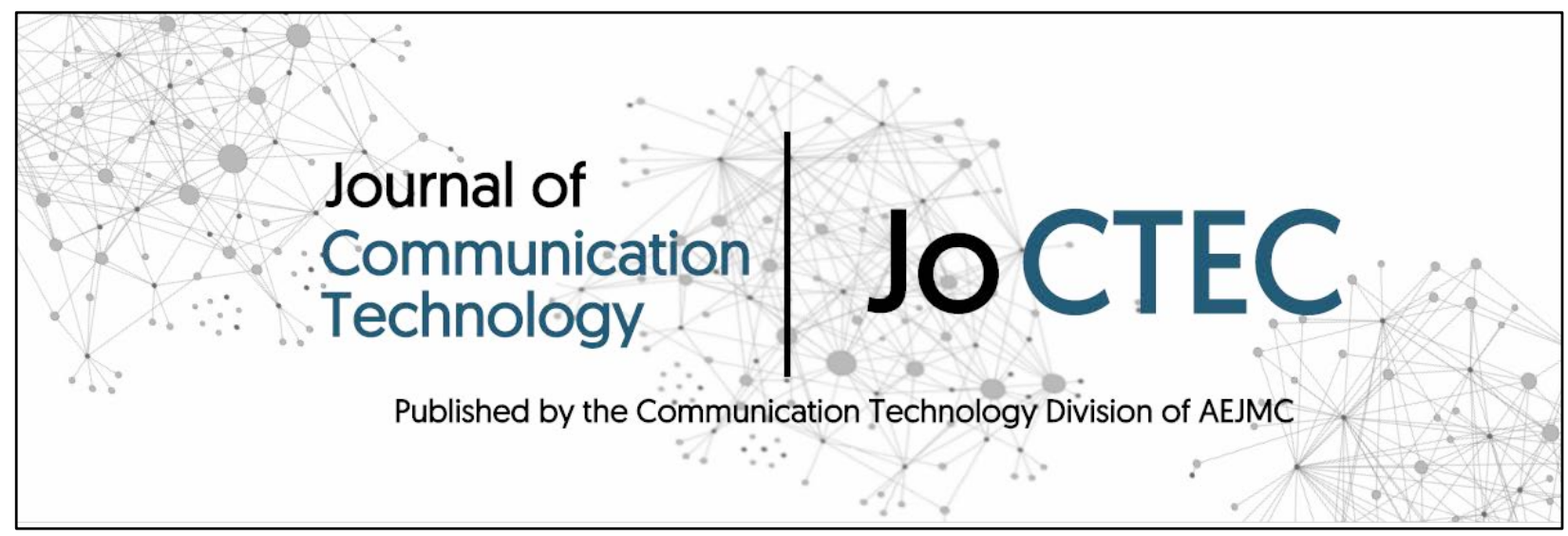

Hey, Nice Run!: Exploring Factors Related to Exercise, Self-Monitoring and Motives for Exercise App Use

\author{
Michael Fuller, Ph.D. \\ Consultant, Tempe, AZ \\ David J. Atkin \\ Professor, Department of Communication \\ Rory McGloin \\ Assistant Professor, Department of Communication \\ University of Connecticut \\ Kimberly Embacher \\ Assistant Professor, Department of Communication \\ Rhode Island College
}

Original manuscript accepted for publication in

Journal of Communication Technology

Published by the Communication Technology Division of the Association for Education

in Journalism and Mass Communication

DOI: 10.51548/joctec-2019-002 
Hey, Nice Run!: Exploring Factors Related to Exercise, Self-Monitoring and Motives for Exercise App Use

\begin{abstract}
The present study tests a multifaceted model that explains exercise app use motives in relation to uses and gratifications as well as emotional and social support factors. A hybrid model based on Uses and Gratifications theory and self-efficacy perspectives was used to examine exercise app use. Gratifications for competition, self-monitoring, and informational and emotional social support were modeled. Structural equation modeling results provide qualified support for a comprehensive path model identifying the role of self-monitoring and personal goal achievement in smartphone exercise app use. The predicted roles for personal goal achievement and exercise app use received support, with personal goal achievement predicting exercise app use. These findings may contribute to the health communication literature through the creation and validation of self-monitoring and exercise app self-efficacy scales.
\end{abstract}




\section{Hey, Nice Run!: Exploring Factors Related to Exercise, Self-Monitoring and Motives for Exercise App Use}

Research suggests that $60 \%$ of all smartphone app users have downloaded at least one mobile health app (Dam, Roy, Atkin, \& Rogers, 2018), the usage of which has increased 330\% from 2015 to 2017 (Netimperative, 2017). Yet, despite the increasing pervasiveness of these apps, the current understanding of user motivations governing their use remains incomplete. Exercise applications offer a wide range of characteristics that users are likely to engage for a multitude of reasons. For example, a majority of the most popular exercise applications (e.g., Fitocracy) have both personal and social elements, which are likely to produce unique motivations for users (Amnesi, 2016). Moreover, these applications also offer multiple levels of feedback, from helping users achieve their goals to challenging them to take their abilities to the next level.

Research on exercise app use has yet to explicate a comprehensive set of motives for their use (e.g., Breton, Fuemmeler, \& Abroms, 2011; Cowan and colleagues, 2013; Krebs \& Duncan, 2015; Patel, Asch, \& Volpp, 2015; Rabin \& Bock, 2011). Furthermore, exercise app use motives may or may not be the same as those governing use of news apps or game-based apps. Given their popularity and unique multi-faceted programming, this exploratory research seeks to examine the specific motivations for engaging with exercise applications. This study proposes an integrated framework that encompasses variegated domains - ranging from digital media to health sciences - to examine exercise app adoption and use. Specifically, the present study advances a comprehensive model based on audience uses and gratifications (U\&G), digital health technology clusters, and self-efficacy and basic needs, as explicated in the emotional gratifications (EGRATS) scale (Strizhakova, Kang, \& Buck, 2007). The latter component 
complements a novel U\&G framework—including competition and self-monitoring — alongside social support and ascriptive factors.

\section{Self-Efficacy}

\section{Literature Review}

Bandura (1986) defines self-efficacy as “people's judgments of their capabilities to organize and execute a course of action required to attain designated types of performances" (p. 391). Self-efficacy plays a critical role in the process of sustained exercise. Individuals who do not believe they have the competency to perform a given behavior-such as eHealth monitoring - are less likely to undertake or sustain that behavior over time (e.g., Lagoe \& Atkin, 2015). Enhancements to task-related self-efficacy can improve one's belief in his or her ability to perform a given task and, hence, achieve a desired outcome. Self-efficacy has been used in health interventions and technology-based research, both of which are relevant to the present discussion, given that exercise apps are health-related and rely on technology to work. Research suggests that improvements in self-efficacy lead to increased exercise, a healthier diet, and weight loss (e.g., Annesi, 2016).

Self-efficacy can thus influence relationships governing the intersection between exercise, competition, and technology use. Exercise history (or experience), which is defined as how long a person has been consistently exercising, is related to exercise self-efficacy. Benisovich, Rossi, Norman, and Nigg (1998), for instance, found that self-efficacy helps to overcome excuses to not exercise; they define exercise self-efficacy as a person's belief that he or she can perform the necessary actions to achieve the goal of exercise performance. Consequently, as exercise history increases - that is to say, the longer a person has been consistently performing a given exercise - the higher their exercise self-efficacy will be (Annesi, 
2016). Self-efficacy has also been related to Internet use (e.g., Eastin \& LaRose, 2000) as well as online information seeking for health engagement (e.g., Lagoe \& Atkin, 2015), using the Internet to monitor physical activity (e.g., Carr and colleagues, 2013), and online search motivations and behaviors (Lin and colleagues, 2015).

Given its importance for Internet skills, self-efficacy appears to play a dynamic role in smartphone app use (e.g., Kim, Jun, Han, \& Kim, 2013) and by extension, smartphone exercise app use. The present study seeks to understand the role of smartphone exercise app self-efficacy, which is defined as "how much a person believes he or she has the ability to operate the functions of a smartphone exercise app." Dam et al. (2018), for instance, found that exercise selfefficacy predicts health app use. One can assume that the self-efficacy dynamics motivating Internet use also obtain for smartphone app adoption. Specifically, self-efficacy — in general and exercise app contexts — should bear positive relations with exercise experience and app use. Drawing from the research and theory reviewed above, then, it is posited that:

H1: Exercise experience will positively influence exercise self-efficacy.

H2: Exercise app self-efficacy will positively influence exercise app use.

Due to the limited research on exercise apps, scholarly work appears to be silent on the relationship between exercise self-efficacy and smartphone exercise app use. Although apps might appeal to fitness enthusiasts, those feeling more efficacious about exercise might feel less need for such devices. Given these countervailing influences, the following research question is proposed:

RQ1: How does exercise self-efficacy influence exercise app use?

\section{Emotional Needs}


Early studies identified emotional needs as a potential gratification for media use, for example, watching TV for emotional release (Katz, Blumler, \& Gurevitch, 1974). In addition, the role of emotions in media use is consistent with the U\&G framework (e.g., Strizhakova et al., 2007). Rosengren's (1974) U\&G process model begins with basic needs - including emotionswhich in turn influence the media selection process. Similarly, Bartsch, Mangold, Viehoff, and Vorderer (2006) found that emotions themselves may be a gratification, which they refer to as emotional gratifications. These "e-grats" ranged from positive/rewarding feelings (e.g., "fun") to more power-based "reptilian" expression of emotions (Strizhakova et al., 2007).

Mood management theory (e.g., Zillmann, 2000) also examines the importance that emotion has in media use. This perspective suggests that a variety of emotions-including negative emotions such as fear and sadness — can lead to pleasure for media users. The key, according to Zillmann, is the role of arousal, which he suggests most users prefer in moderate amounts for a satiated state. Building on mood management research, Strizhakova et al. (2007) found emotion to be a powerful driver of media selection, notably the sad versus happy contrast. Given that past research extends work on media use motivations to the parallel realm of mobile media (e.g., Wei, Karlis, \& Haught, 2012), one can assume that these commonalities could extend to the realm of health app uses. Based on the theory and research reviewed above establishing the importance of emotion as a determinant of media use, it is posited that:

H3: Emotional needs will positively influence motives to use smartphone exercise apps.

\section{Uses and Gratifications Theory}

$\mathrm{U} \& \mathrm{G}$ theory identifies the reasons why people use mass media, suggesting that such use meets a variety of psychological and social needs (Katz, et al., 1974). U\&G assumes that the strength of one's motivation determines media use, which is goal-directed. This assumption 
works well with interactive media such as the Internet, computers, mobile phones, and smartphones (Sundar \& Limperos, 2013). The perspective is thus well-suited to explain the needs and gratifications sought by users - as well as the results of their behavior (Rubin, 2009; Lin, 2009; Palmgreen, Wenner, \& Rosengren, 1985) —informing the app uses that we explore in turn.

Smartphone apps. Recent U\&G research examining apps has identified constant availability as a specific gratification related to smartphone usage (Wei, et al., 2012). Similarly, convenience was found to be an important gratification of smartphone use (Wei et al., 2012; Weiss, 2013). Additional smartphone gratifications have also been identified, such as entertainment and information seeking (Wei et al., 2012; Weiss, 2013). Building on this work, the present study proposes four motives for exercise app use-self-monitoring, competition, informational social support, and emotional social support—which are described below.

Self-monitoring. This concept describes the process whereby participants monitor some type of health parameter or outcome. For example, Helsel, Jakicic, and Otto (2007) found the process of self-monitoring to be important in assisting with physical activity, weight loss, and changing eating habits among diabetics. Similarly, Ayabe and colleagues (2010) found that patients with chronic disease conditions increased their physical activity as they monitored the amount of time they spent exercising. Carels and colleagues (2005) found that consistent selfmonitoring of exercise was associated with greater exercise and weight loss.

Smartphone exercise apps may provide benefits for users who want to monitor exerciserelated metrics, such as the distance, speed, and time of a workout, while also being able to save the workout and review it in the future (Rabin \& Bock, 2011). Additional benefits of using exercise apps may include 24-hour access to exercise tracking and health information. Many 
apps also offer online support communities where users can post their workouts and receive feedback from other users (Carr et al., 2013). Further, exercise apps have the ability to provide a more individualized user experience and facilitate greater ease in self-monitoring. This may lead to higher levels of self-efficacy, leading to improved health outcomes (e.g., Lin et al., 2015). Lastly, exercise apps provide the ability to self-monitor and obtain health and exercise information, independent of location (Breton et al., 2011).

Self-monitoring capabilities of exercise apps not only benefit the overweight and obese population, but also those who exercise consistently, and meet or exceed the exercise amount recommended by the Centers for Disease Control (Fogg, 2003). Most exercise apps allow for the tracking or recording of exercise-specific information identified by Augemberg (2012), such as distance covered, time spent exercising, pace/rate/speed, and (with the appropriate sensors) other metrics like heart rate. Fogg (2003) contends that for a technology to be effective for selfmonitoring, it should work in real time, giving users immediate and engaging feedback related to their progress on a particular goal or task. Annesi (2016) argues that digitization allows the process of data collection to be automated, thus freeing users of the tedious burden of monitoring their exercise. Rabin and Bock (2011) found that the ability to track exercise was very important to app users. Consequently, the above theory and findings underscore the importance of selfmonitoring for exercise app use. More formally, it is posited that:

H4: Self-monitoring of health behavior will positively influence exercise app use.

Competition through media. Competition emerged as an important motive in listening to radio game shows (Katz et al., 1974). Competition through exercise app use can occur in many types of exercise apps, particularly those that gamify exercise. Users of these apps can earn points for completing challenges and for a session of exercise. A user's most recent exercise and 
point total are posted in the feed-similar to the news feed in Facebook-making this information visible to other users (see Figure 1). Thus, as users complete exercise, achieve challenges, and advance in level, this information is posted in the feed, thus facilitating competition.

Masters and Ogles (1995) found competition to be an important motive for marathon runners with differing experience levels. Their findings suggest those running three or more races indicated competition as a key motive, while runners completing only two races were motivated by performance improvement. This may indicate that those who have been exercising longer could identify competition as a stronger motive than less experienced exercisers. LaChausse (2006) found that motives for cyclists differ based on gender; men were more likely to be motivated by competition than women.

Similarly, Ingledew and Markland (2008) found competition to be an important motive among office workers who were involved in a work place-based exercise program. Dam et al.'s (2018) study of health app adoption found support for a novel gratification involving competitive challenges with other users. One can assume that the competitive dynamics outlined above with health apps and other digital media will apply to exercise app use. Based on the findings and theory reviewed above, it is posited that:

H5: Competition motivation will positively influence exercise app use.

\section{Informational and Emotional Social Support}

House (1981) defined emotional social support in the context of actions that convey esteem. Examples of emotional support include "statements of affection, emotional understanding, and statements geared towards relieving pain and stress" (Walther \& Boyd, 2002, p. 6). This type of support may be important to exercise app users, especially among those 
struggling with exercise, as an encouraging comment or gesture may be all that is needed to provide support. This might include, for instance, online congratulations offered by a follower in response to an exercise achievement (See examples in Figures 3-5).

Scholars (e.g., Dam et al., 2018; House, 1981) suggest that informational support (i.e., advice or information that facilitates problem solving or competition) is a form of social support. Walther and Boyd (2002) found informational support to be an important online activity, one made possible by the ability to perceive or provide support anytime. Exercise app research has also identified the importance of users being able to communicate with each other to gain social support (Breton et al., 2011) and to comment on and follow other users' workouts (Carr et al., 2013). Based on the theoretical dynamics governing informational and emotional support outlined above, one can assume that online support encourages use of exercise apps in pursuit of one's fitness goals; more formally:

H6: Informational social support will positively influence exercise app use.

H7: Emotional social support will positively influence exercise app use.

\section{Technology Clusters}

Rogers (2003) identifies technology clusters as elements of a technology that users perceive as being related, like wireless connectivity of a laptop and mobile phone. He further illustrates how technologies that are adopted during the same time period are often symbiotic in nature (Rogers, 2003). Thus, the more technological devices a person owns, the more likely they will adopt new innovations.

Use of technologies that are functionally similar has been shown to predict adoption and use of a given modality (Atkin, 1993). For example, Leung and Wei (1998) examined the use of technology clusters in the context of mobile phone adoption and found those owning fewer 
technologies were less likely to adopt mobile phones. In addition, Lin (2009) identified a positive role of technology clusters in predicting the adoption of online radio. Finally, Hunt, Lin, and Atkin (2014) found that technology clusters predicted the use and frequency of sending photo messages. Consequently, technology clusters are likely to influence the use of exercise apps. Rogers (2003) argues that when an innovation is adopted, technologies are often interdependent. To wit, those using exercise apps may also use other exercise-related technologies. Relevant exercise-based technologies residing in a proposed exercise technology cluster might, for instance, include: a GPS-based watch such as a Garmin, Nike, or Timex; a wearable activity monitor such as a Fitbit or Jawbone; a pedometer; and/or a heart rate monitor. Assuming that technology cluster dynamics can apply to media as well as exercise contexts, it is posited that:

H8: Technology clusters will positively influence exercise app use.

H9: Exercise technology clusters will positively influence exercise app use.

\section{Narcissism}

Parallel research examining social networking sites has studied the role played by personality dimensions such as narcissism, which could factor into motives for using social networking services (SNS). For example, Krishnan and Atkin (2014) found a sub-dimension of narcissism — vanity — to play a significant role in SNS usage. This suggests that SNS usage fulfils the need for social approval, which is important for those who are vain. Buffardi and Campbell (2008) found narcissism to be related to the number of friends and the number of wall posts between friends through SNS usage. The number of wall posts is illustrative of a technique whereby users can receive social approval, which is important for narcissists (e.g., Ames, Rose, \& Anderson, 2006). 
A key assumption underpinning these links with narcissism suggests that users consider telematic channels, particularly health apps, as useful vehicles for projecting a favorable image of their health (e.g., McGloin, Embacher, \& Atkin, 2017). Embacher Martin, McGloin, and Atkin (2018), for instance, found that appearance-related motivations positively predicted use of calorie-tracking app use among college students. Given the functional similarities between exercise apps and SNS - insofar as both modalities allow users to create a profile, post pictures, upload workouts, and comment on the workouts of friends - it is posited that:

H10: Narcissism will positively influence exercise app use.

\section{Well-being}

The final component of this model involves measures of well-being, which have shifted from a negative, disease-based approach to a positive outcome approach (Bann et al., 2012). Given the importance of regular exercise, it is plausible that exercise app use will be positively related to physical well-being. That said, exercise apps may be sought as a remedy by those in poor health. Owing to the scarcity of work on exercise app use in this context, the following inquires:

RQ2: What is the relationship between exercise app use and well-being?

\section{Process Model}

An integrated perspective on the app use model is proposed in Figure 1. The crux of this model involves the $U \& G$ process, which is identified by the three light blue boxes. This process begins with basic needs, as outlined by Rosengren (1974), which is measured by the emotion variables contained in the EGRATS scale (Strizhakova, et al., 2007). To recap, basic needs can include a variety of psychological and biological needs, including emotional needs. These needs 
can, in turn, influence the motives or the reasons a user decides to use a specific medium or app. The path from basic needs to motives is posited under Hypothesis 3.

The motives variables are identified by the second light blue box in the U\&G process, underpinning the primary study focus on why apps are selected for media use. Motives vary at the individual level and by the type of media selected; motives then drive media use, identified by the final light blue box in the U\&G process (e.g., Atkin, Hunt, \& Lin, 2015). Summarizing the expectations from above, the path from motives to media use is identified in Hypothesis 4-7. Extending from the U\&G process is the path from the media use variable to the health outcome variable (identified as RQ2), which attempts to connect media use to well-being.

Exercise experience, in the purple box, predicts exercise self-efficacy (H1), in the red box. In turn, the influence of exercise self-efficacy on exercise app use is identified by RQ1, while the path from exercise app self-efficacy predicts exercise app use (H2). Additionally, diffusion variables (the lavender box; H8 and H9) will directly influence exercise app use. Finally, narcissism will predict exercise app use (H10).

\section{Methodology}

Study data was obtained through an IRB-approved questionnaire and administered electronically through the Qualtrics survey-hosting site. Participants $(N=393)$ were recruited from a large lecture communication course in which research credit was provided for participation. Given that 18-29-year-olds are among the most avid users of health apps (Netimperative, 2017; Weiss, 2013), this student-based sample was both convenient and purposive. Boulianne's (2015) meta-analysis of current research related to social media use found that, while there is potential for the use of student samples to have a negative effect on the statistical significance of findings, the use of such samples in social media research has been 
quite popular. Further, Mullinix, Leeper, Druckman, and Freese (2015) found evidence for considerable similarity between effects from survey research conducted with student-based convenience samples and nationally representative samples, providing additional support for the use of a student-based sample in the current study. The majority of participants in the sample for the current study were female $(52.7 \%)$ and 19 years old. "Caucasian" was the most prevalent ethnic background category selected by study participants (73\%), followed by Asian (14.8\%), Hispanic (5.1\%), African American (3.3\%), and Mixed Race (2\%), with the remaining 2\% coming from American Indian, Other, and Pacific Islander categories. Average annual household income was $\$ 100,000$.

The study focused on exercise apps, as opposed to dieting apps such at MyFitnessPal. Sample participants used smartphone exercise apps an average of two to three times a month ( $M$ $=2.48, S D=1.50)$. Each use lasted an average of just over thirty minutes $(\mathrm{M}=2.46, \mathrm{SD}=1.40)$. Fitness apps $(M=3.68, S D=2.20)$ and running apps $(M=3.44, S D=1.90)$ were the most popular, followed by walking apps $(M=2.60, S D=2.0)$ and cycling apps $(M=1.95, S D=1.50)$.

\section{Measures}

Competition. Three different measures were utilized, the first two aimed at measuring competition through physical activity and the last one measuring competition through media. The first scale was adapted from the competition subscale of the Exercise Motivations Inventory from Markland and Ingledew (1997) $(\alpha=.93, M=4.22, S D=1.40)$ and had a response range from 1 (strongly disagree) to 7 (strongly agree). This scale contains four motivation items, including "because I enjoy physical competition." The second scale, video game competition, was taken from Sherry and Lucas (2003) and consisted of four items $(\alpha=.88, M=3.77, S D=$ 1.36). The scale had a response range from 1 (strongly disagree) to 7 (strongly agree). This 
includes such items as: "I like to play to prove to my friends that I am the best." The last scale examines competition with self, or as identified by Masters and Ogles (1995), personal goal achievement. The seven-point scale consists of six items $(\alpha=.93, M=4.98, S D=1.35)$, including such motivations as "To make my body perform better than before."

Informational and emotional social support. Both seven-point scales were taken from Choi and Chen (2006) and contain three items. Scale reliabilities were acceptable for both informational $(\alpha=.85, M=3.98, S D=1.40)$ and emotional social support $(\alpha=.79, M=3.60, S D$ =1.38). Participants were asked "Why do you use exercise apps?" and were provided support responses including "Seek suggestions about how to deal with a personal problem." The emotional social support component included such items as "Seek encouragement."

Exercise app self-monitoring scale. A new scale was created to measure the importance of being able to track one's progress while exercising through the use of an exercise app. This seven-point general self-monitoring scale included three items for measuring self-monitoring, including "I like to see how far I've gone." A confirmatory factor analysis (CFA) was run on the exercise app self-monitoring scale in AMOS 22. Results indicated a solid model fit (RMSEA = $.05)$, and alpha reliability was high $(\alpha=.85, M=5.55, S D=1.16)$.

EGRATS scale. This seven-point scale was adapted from Strizhakova et al. (2007), as it represents basic human emotions, regardless of context. The EGRATS scale had 38 items, of which 18 were relevant to the present study. Participants were asked "I feel after I have engaged in physical activity" for all 18 items, which comprise six subscales.

The negative prosocial scale included "Ashamed," "Embarrassed," and "Guilty" $(\alpha=.82$, $M=2.10, S D=1.10)$. The negative individualism scale included "Angry" and "Arrogant" $(\alpha=$ $.72, M=2.50, S D=1.35)$. The positive individualism scale included "Happy," "Satisfied," and 
"Confident" ( $\alpha=.88, M=5.60, S D=1.06)$. The positive prosocial scale included "Proud" and

"Triumphant" ( $\alpha=.70, M=5.10, S D=1.30)$. The reptilian sex scale included "Erotic,"

"Aroused," and "Sexy" $(\alpha=.82, M=3.14, S D=1.50)$.

The reptilian power scale included "Vigorous," "Energetic," and "Powerful" $(\alpha=.67, M$ $=4.98, S D=1.13)$. A CFA was conducted and indicated that "Vigorous" had a low factor loading of .505, while "Powerful" and "Energetic" had much higher factor loadings, .895 and .894 respectively. "Vigorous" was removed from the scale, which improved model fit to $\mathrm{RMSEA}=.078, \mathrm{CFI}=.942$, and a reliability of $\alpha=.84(M=5.40, S D=1.20)$.

Exercise technology cluster. Participants were asked which of the following items they use on a rating scale ranging from 1 ("never") to 5 ("frequently"). The prompt asked "Which of the following items do you own?" and was followed by "a GPS based watch such as a Garmin, Nike or Timex," “a Fitbit or Jawbone," "a pedometer," and "a heart rate monitor." The scale reliability was $\alpha=.80(M=1.34, S D=0.65)$.

Technology cluster. Original technology cluster scales were proposed. One item asked "Which of the following items do you own?" Answers included: "A laptop," "a tablet device such as an iPad," “an e-reader such as a Kindle," and "a digital audio player such as an iPod," and participants were instructed to select 0 for an item they do not own and 1 for an item they do own. The scale's reliability was poor, $\alpha=.25(M=3.10, S D=0.65)$, necessitating its removal from the analysis.

Exercise app use. This scale contained seven items, which were summed to form a composite scale. Question 1 asked participants "How often do you use exercise apps?" with the response set: Once per month; 2-3 times per month; Once per week; 2-3 times per week; 4-5 times per week; 6 or more times per week. Question 2 asked participants "How much time do 
you use an exercise app each session?" with the response set: 0-15 minutes; 16-30 minutes; 3145 minutes; 46-60 minutes; 1-2 hours; 2 hours or more. Questions 3-6 asked participants how often they used the following exercise app types "Running App," "Cycling App," "Walking App," and "Fitness App" with the response set: Never, Several Times a Year, Once a Month, 23 Times a Month, Once a Week, 2-3 Times a Week, 4-6 Times a Week, and Daily $(\alpha=.75, M=$ $16.60, S D=7.10)$.

Narcissism. The Narcissism Personality Index-16 (NPI-16; Ames et al., 2006) was adapted here as a shortened version of the NPI-40 (Raskin \& Terry, 1988). The 40-item scale has better reliability ( $\alpha=.83$ compared to .70 ) than the 16-item scale. Due to the length of the survey, the risk of participant fatigue was believed to be a greater threat to this study. Consequently, the 16-item scale was used. However, the NPI scale produced a weak alpha of .42, prompting its removal from the analysis due to poor reliability.

Well-being scale. This scale was taken from the Public Health Surveillance Well-Being Scale, developed for the CDC (Bann et al., 2012). The 10-item scale asked participants to indicate "How satisfied you are with each of the following items" on a scale from 1 (strongly disagree) to 5 (strongly agree), and included such items as "I am satisfied with my life." The well-being response domains ranged from "your family life," and "your social life" to "your energy level." The scale alpha reliability was .70 $(M=3.10 ; S D=0.69)$.

Exercise self-efficacy scale. This scale contained six subscales with three items each and was derived from Benisovich et al. (1998). Participants were asked how confident they were in their ability to exercise under a specified condition. The responses ranged from 1 (not at all confident) to 5 (completely confident). The negative affect subscale included items such as "I am anxious" $(\alpha=.82, M=2.72, S D=0.95)$. Excuse making, the second subscale, included items 
such as "I am busy" ( $\alpha=.86, M=2.30, S D=0.96)$. The exercise alone subscale included items such as "I am alone" $(\alpha=.86, M=3.27, S D=1.1)$. The inconvenient to exercise subscale included items such as "I don't have access to exercise equipment" $(\alpha=.85, M=2.48, S D=$ 1.02). Resistance from others, the fifth subscale included items such as "My friends don't want me to exercise" $(\alpha=.87, M=2.87, S D=1.10)$. The last subscale, bad weather , included items such as "It's cold outside" $(\alpha=.94, M=2.68, S D=1.16)$.

Exercise app self-efficacy scale. This scale was adapted from Lin et al. (2015) and included four items, such as: "I am confident in my ability to use smartphone exercise apps." Responses ranged from 1 (strongly disagree) to 7 (strongly agree), and the scale had high reliability ( $\alpha=.94, M=5.40, S D=1.25)$. Finally, social locators were used to profile the sample and were measured with commonly accepted measures. Gender, for instance, was dummy-coded (1 if male).

\section{Data Analysis}

Following data collection, CFA was performed on each measure using AMOS 22.0. Palmgreen et al. (1985) reasoned that-second generation multivariate modeling techniques—such as structural equation modeling (SEM) - allowed for more comprehensive testing of complex U\&G models (relative to "first-generation" multivariate procedures like multiple regression and canonical correlation analysis). Hypothesis and research question testing were thus performed using SEM in AMOS 22, utilizing maximum likelihood estimation. The procedure used in this study for structural equation modeling is discussed in turn.

\section{Results}

\section{SEM Procedure}


Measurement model. Following CFA, the scales were examined to determine if items needed to be trimmed, which involves removing items that load poorly on the underlying factor. If the measurement model indicated a good fit, the analysis moved onto the structural model, where relationships between variables were analyzed using structural equation modeling.

Structural model. The structural model tested the hypothesized relationships among variables, as indicated in Figure 1. The structural model was examined for goodness of fit (Holbert \& Stephenson, 2003), after which the model was re-specified. This involved removing non-significant paths from the structural model and, when necessary, adding theoretically justified paths that were not included in the initial hypothesized model.

\section{Hypothesis and Research Question Testing}

Hypothesis 1, which predicted that exercise experience would be positively related to exercise self-efficacy, received partial support, with exercise experience predicting the exercise alone subscale of exercise self-efficacy $(\beta=.32, p<.001)$. Exercise app self-efficacy approached significance as a predictor of exercise app use $(\beta=.10, p=.053)$, leaving Hypothesis 2 without support. Because of the non-significance and small effect on exercise app use, exercise app selfefficacy was removed from the model, which also obviated the need to test Research Question 1. Hypothesis 3 predicted that emotion would be positively related to motives for exercise app use; the hypothesis received partial support, as positive individualistic emotion and reptilian power predicted self-monitoring $(\beta=.34, p<.001$ and $\beta=.17, p<.001$, respectively). Hypothesis 3 also received additional support, with positive prosocial emotion predicting personal goal achievement $(\beta=.23, p<.001)$.

Self-monitoring did not predict exercise app use $(\beta=.03, p=.46)$, failing to provide support for Hypothesis 4 and thus necessitated the removal of the self-monitoring variable from 
the model. However, this variable was re-conceptualized as a perceived solution variable based on the modification indices in AMOS and based on theory (e.g., Rosengren, 1974).

Consequently, self-monitoring was moved so that it followed emotion variables (as initially proposed) and preceded motives (differing from the hypothesized model)—specifically, goal achievement. Self-monitoring in this new location significantly predicted personal goal achievement $(\beta=.53, p<.001)$, providing partial support for Hypothesis 4 .

Focusing on motives, competition did not predict exercise app use $(\beta=.04, p=$ .49), leaving Hypothesis 5 without support. Consequently, competition was removed from the model, along with the reptilian sex and reptilian power paths that led to competition. The personal goal achievement motive did, however, predict exercise app use $(\beta=.34, p<.001)$, providing partial support for Hypothesis 5. Informational social support failed to predict exercise app use $(\beta=.02, p=.73)$, thus Hypothesis 6 was not supported. Informational social support was thus removed from the hypothesized model. Emotional social support was also unrelated to exercise app use $(\beta=-.001, p=.98)$, thus Hypothesis 7 was not supported. Emotional social support was thus removed from the hypothesized model. The predicted relationship involving technology clusters (Hypothesis 8) could not be tested, since the measure was dropped due to the aforementioned reliability issues, as was the case with narcissism (Hypothesis 10). Hypothesis 9, which predicted that exercise technology clusters would predict exercise app use, received support $(\beta=.32, p<.01)$. Despite the support observed here for several hypothesis and research questions, the initial hypothesized model (see Figure 1) had a poor fit, with an RMSEA of .132 and CFI of .369. The model was re-specified by deleting statistically non-significant paths and non-significant variables and adding theoretically or 
conceptually justified paths. Through this process of re-specification, the final model attained good fit, with CFI $=.968$, RMSEA $=.052, \chi^{2}=49.90, d f=24, \mathrm{p}<.001$ (see Figure 2$)$.

Owing to the deleted portions of the model mentioned above, tests involving the Research Questions — regarding the influence of self-efficacy (RQ1) and well-being (RQ2) on exercise app use-were not statistically differentiated. With regard to other findings, a positive relationship emerged between male gender and self-monitoring, $\beta=.15, p<.001$. In addition, the exercise alone scale predicted exercise app use $(\beta=.14, p<.001)$.

\section{[INSERT FIGURE 2 ABOUT HERE]}

The remaining four subscales of the exercise self-efficacy scale on exercise app use were not significant. These included the negative affect sub scale $(\beta=.009, p=.84)$, excuse making subscale $(\beta=-.024, p=.60, \mathrm{~ns}$.$) , inconvenient to exercise subscale (\beta=.036, p=.41)$ and resistance from others subscale $(\beta=-.013, p=.77)$.

\section{Discussion}

The present study extended work on general app use (e.g., Wei et al., 2012) to smartphone exercise apps. Given the interdisciplinary scope of this investigation, a hybrid model entailing $U \& G$ theory, exercise self-efficacy, and diffusion of innovations was used to examine exercise app use. The innovation of this approach involves the application of motives for smartphone exercise app use in light of motivations encompassing exercise competition as well as media U\&G. This study also contributed to the literature through the creation of selfmonitoring and exercise app self-efficacy scales.

Study results provide qualified support for a comprehensive model explaining app use in terms of media/exercise motivations and psychological factors like self-efficacy. Per the latter, the predicted path received partial support, with exercise experience predicting the exercise alone 
subscale of the self-efficacy variable. Thus, the more exercise experience that a person has, the more likely they are to exercise, even if this means they must exercise alone. Consequently, a person with extensive exercise experience will not let exercising alone be a deterrent to accomplishing their goals. This finding seems consistent with the literature on self-efficacy (e.g., Bandura, 1997).

The hypothesized influence of exercise app self-efficacy and exercise app use, however, failed to receive support. This seems surprising, given the results of similar research have found self-efficacy to be important in media use (e.g., Kim et al., 2013), particularly for health information (Lagoe \& Atkin, 2015). One possible explanation for why exercise app self-efficacy may not be relevant to those who use exercise apps could stem from the relative maturity of this innovation; that is, participants of this study know how to use smartphone apps, rendering the relationship between self-efficacy and exercise app use moot as the practice becomes normalized.

By contrast, emotional needs and exercise app use models received partial support, as positive prosocial emotion predicted personal goal achievement. While self-monitoring was initially conceptualized as a motive, it was later re-conceptualized as a perceived solution variable. Because of the initial conceptualization of self-monitoring, the role of emotion in selfmonitoring is considered in the present model. Specifically, positive individualistic and reptilian power (i.e. primal) emotions predicted self-monitoring. This dynamic underscores the apparent importance of (self) impression management goals in determining exercise app use.

The predicted influences of self-monitoring and exercise app use did not, however, receive support. That said, modification indices for the hypothesized model suggest that selfmonitoring should precede the motive variables, specifically a direct path to personal goal 
achievement. Upon closer examination, the self-monitoring variable appears to fit the description of what Rosengren (1974) described as a perceived solution variable; that is, simply a way of solving a problem, acknowledging that a problem(s) can be solved in other ways besides media use. In particular, the perceived solution variables should follow basic need variables and precede motive variables. Conceptually, moving the self-monitoring variable between the emotion variables and the motive variables made sense and was theoretically justified.

Extending the study focus to functionally similar media, video game use-like competition and exercise app use - failed to predict app use. One possible explanation for this may stem from the structure of exercise apps, some of which could more closely resemble video games and have a more game-like interface. Others apps may be less "gamified," such as those exercise apps that simply track exercise. This study did not differentiate between the various exercise app types, which might represent a useful next step in later work.

More generally, the competition and exercise app use models attempted to extend past findings (e.g., Ingledew \& Markland, 2008; LaChausse, 2006; Masters \& Ogles, 1995; Markland \& Ingledew, 1997), which found competition to be important for participants of such endurance sports as running and cycling, to the use of smartphone exercise apps. This hypothesis also failed to receive support. One potential explanation for the inability to replicate those results may involve the difference in samples, as the general student population queried here may have had fewer endurance sports participants than could be found in more purposive samples in earlier studies (e.g., tri-athletes).

By contrast, the predictive roles for personal goal achievement and exercise app use received support, with personal goal achievement predicting exercise app use. This result appears to be consistent with the findings of Masters and Ogles (1995) and extends the use of their 
personal goal achievement scale to smartphone exercise app use. These findings also support the contention of early mass media researchers - suggesting that media users were aware of their media use and that media use was goal driven (e.g., Rosengren, 1974; Katz, et al, 1974)—as well as more recent work focused on app use (e.g., McGloin, et al., 2017, Embacher Martin et al., 2018). Later work might profitably apply less media-centric motivational perspectives — such as the "perceived utility" concept from the technology adoption model (TAM) — to the issue of app adoption.

Moving beyond media use motivations, also examined were models specifying the influence of informational social support on exercise app use and emotional social support and exercise app use. Both failed to receive support. One potential explanation may stem from this study not fully differentiating the types of exercise apps. Some apps offer more of social network site interface and feel, such as Fitocracy (see Figure 5). Therefore, certain apps may be more suited for providing informational social support and emotional social support, when compared to other exercise apps that are used predominantly for exercise tracking.

In addition, the positive influence posited for updated technology cluster on exercise app use was unsupported. The poor scale reliability observed for the updated technology cluster scale, which necessitated the scale's removal from the study, may stem from the range restricted ("yes/no") technology ownership categories. Moreover, the items contained within the technology cluster scale may not have been relevant to a study of exercise apps. Perhaps generalized measures of individual technologies are poor discriminators as digital technologies become normalized. The expectations concerning exercise technology clusters and exercise app use did, however, receive support. This result suggests that, as technology becomes increasingly 
specialized, technology clusters should be updated to reflect this specialization (see Atkin et al., 2015; Wei, 2013).

More broadly, poor fits can occur not only because of responses that are not expected, but also because of errors in data approach and item mixing. These limitations can be a function of the sheer volume of data collected and the ambitious set of testing items. The posited relationship involving exercise app use also did not receive support, while narcissism could not be assessed. The narcissism scale had poor alpha reliability and was dropped from the analysis. This poor reliability may stem from the use of the Narcissism Personality Index-16 (NPI-16) from Ames, et al. (2006), a shortened version of the NPI-40 (Raskin \& Terry, 1988); the shorter scale employed here was less robust than the latter scale. Although the 16-item scale was selected to limit participant fatigue, future research may be more successful in applying the full scale in this context.

The study expectation that exercise self-efficacy would increase exercise app use received partial support. Five subscales_-bad weather, inconvenient to exercise, negative affect, excuse making, and resistance from others-did not significantly predict exercise app use. Must exercise alone did, however, predict exercise app use. The significance of this subscale may stem from those who exercise alone and thus take their phone in case of an emergency or getting lost. If a user has a phone with them, they would logically track their exercise owing to the availability of the exercise app. Another possible explanation is that those who exercise alonefor whatever reason (e.g., exercise partner is too slow/fast) — use exercise apps to track their goals, consistent with the current findings.

On balance, the findings provide important implications for health care practitioners. The relatively stronger explanatory power of attitudinal measures (e.g., exercise motives) - 
particularly in relation to social locators — suggests the importance of perceived utilities served by apps (as opposed to primary diffusion related variables like socio-economic status). This profile seems consistent with that of a more mature medium, where initial adoption is less critical than successful reinvention and maintenance of an innovation. App designers may be wise to consider emerging media use motivations that extend beyond traditional media (e.g., information) and extend to mobile contexts (e.g., "coolness"; see Charney \& Greenberg, 2002). Given the social support functions of app use uncovered in past work (e.g., Dam et al., 2018), designers would be wise to consider visually compelling interfaces that enable users to post and react to information posted by users for public comment (per Figure 4). More specifically, the platform should provide users an easy way to congratulate their peers or to share their performance metrics using visually driven data displays.

An important limitation of this study involves the student-based sample, even though college students represent an important target for technology use and are heavy users of apps (see McGloin et al., 2017; Embacher Martin et al., 2018; Weiss, 2013). Admittedly, the sample obtained in this manner is not representative of the U.S. population selected through traditional random sampling techniques. Hence, caution should be exercised in interpreting results, and future research should look to approach a larger, more generalizable audience. Later research should also investigate apps that allow users to track and self-monitor their exercise automatically, such as Strava and Map My Run, compared with those that require the user to manually enter their exercise information once complete, such as Fitocracy. As media convergence continues to blur the distinctions between media platforms (e.g., Wei, 2013), later work should continue to blend perspectives based on $U \& G$ - or related motivational frameworks 
(e.g., the Technology Adoption Model; see Atkin et al., 2015)—with exercise motivation research. 
Figure 1. Comprehensive research model, which had poor fit, $\mathrm{RMSEA}=.132, \mathrm{CFI}=.369$.

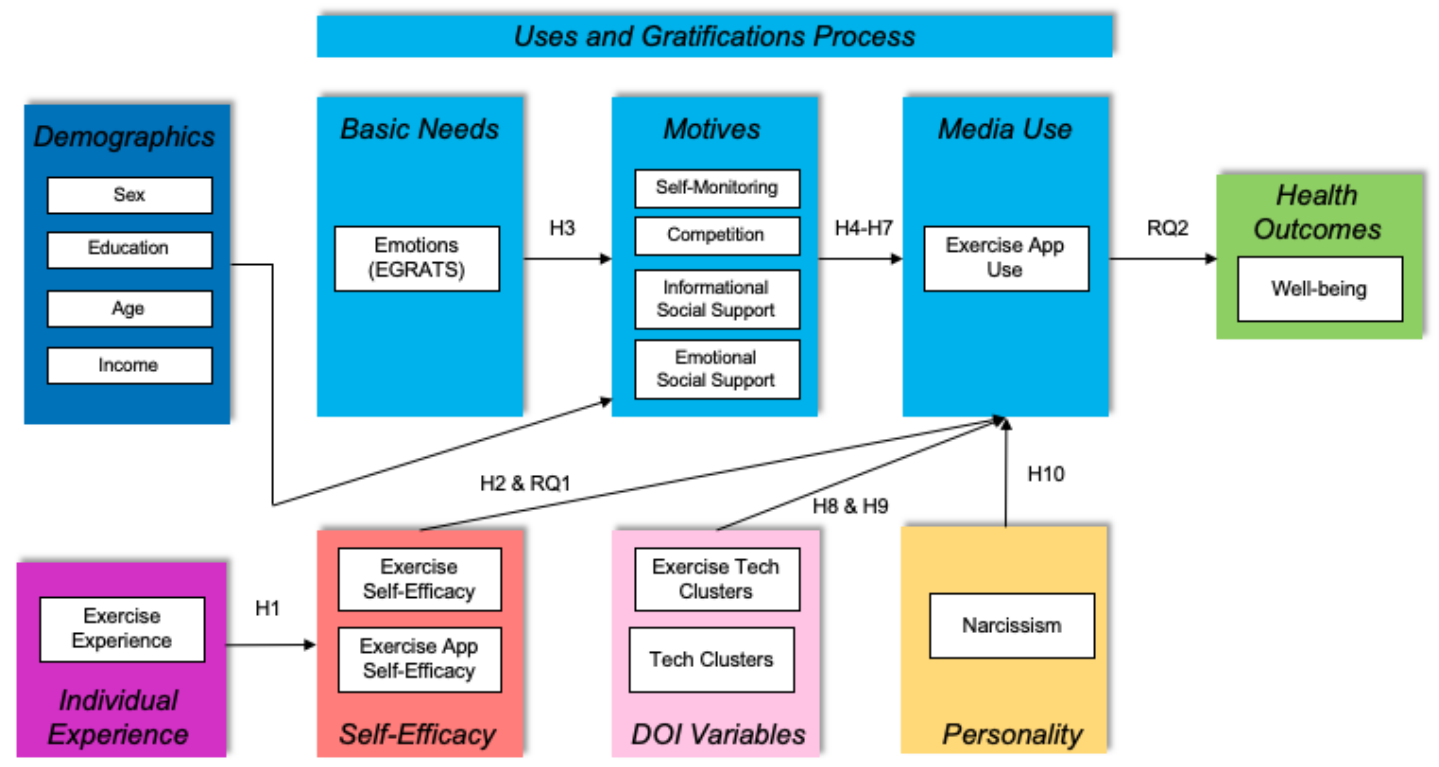

Figure 2. The re-specified model indicated strong measures of fit, CFI $=.968, \mathrm{RMSEA}=.052$, $\chi_{2}=49.90, d f=24, p=.001$. All paths significant at $p<.001$, except $*$ where $p<.05$.

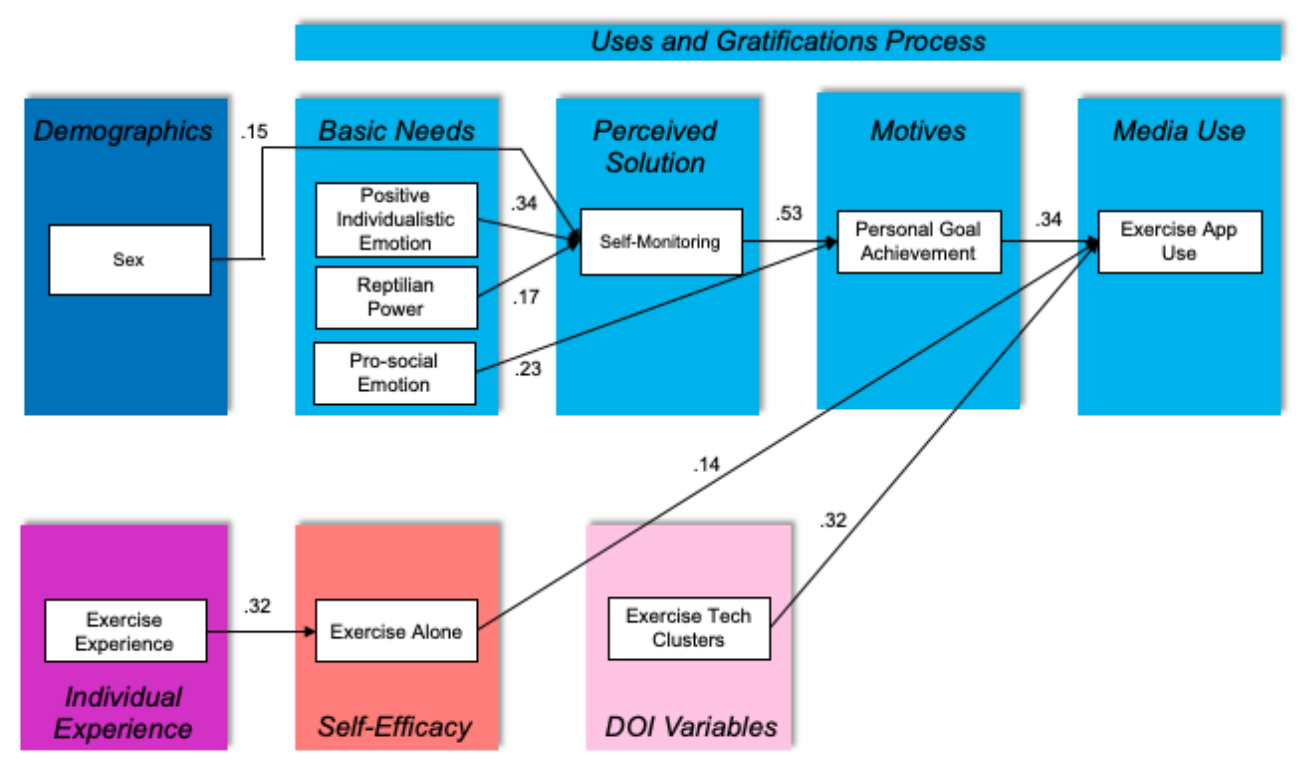

Figure 3. A screenshot of Fitocracy, illustrating the game like interface with Level and point total. Notice the most recent workout indicating the user just earned 1,093 points. 


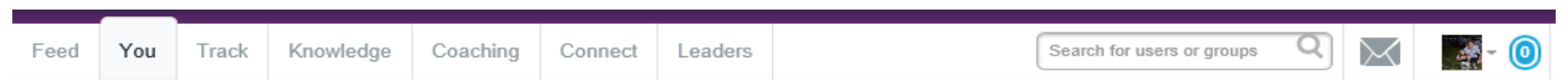

\section{FITOCRACY.}

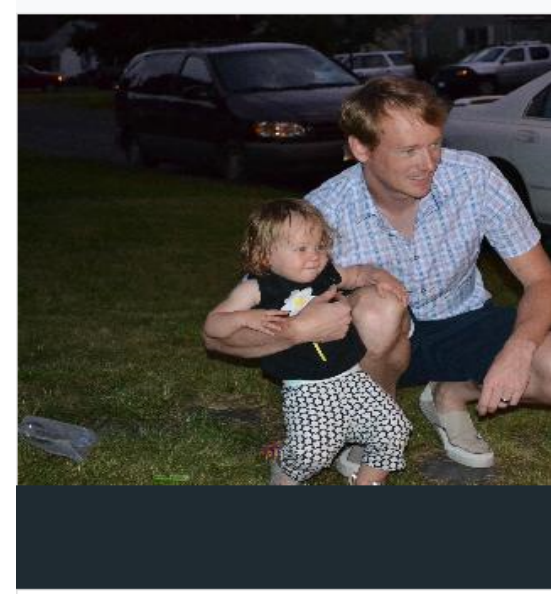

\section{bike2run}

6475 pts to Level 36

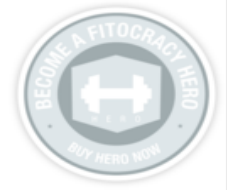

Husband, father, student, triathlete and mountain

biker.

Read More

\section{5 \\ 265,875 \\ 6' 2" \\ $\boldsymbol{\mathcal { O }}_{\mathrm{den}}^{\mathrm{een}}$}

About

Feed

Looking good todav! Tell us what vou're up to.

\%

Activities

Figure 4. A screenshot of the Fitocracy App, showing the achievements this user has earned. Some are related to the use of the app, such as completing their profile. While others are based on exercise, for example, the Hallowed Harrier achievement indicates the user has run $1000 \mathrm{~km}$ in their lifetime.

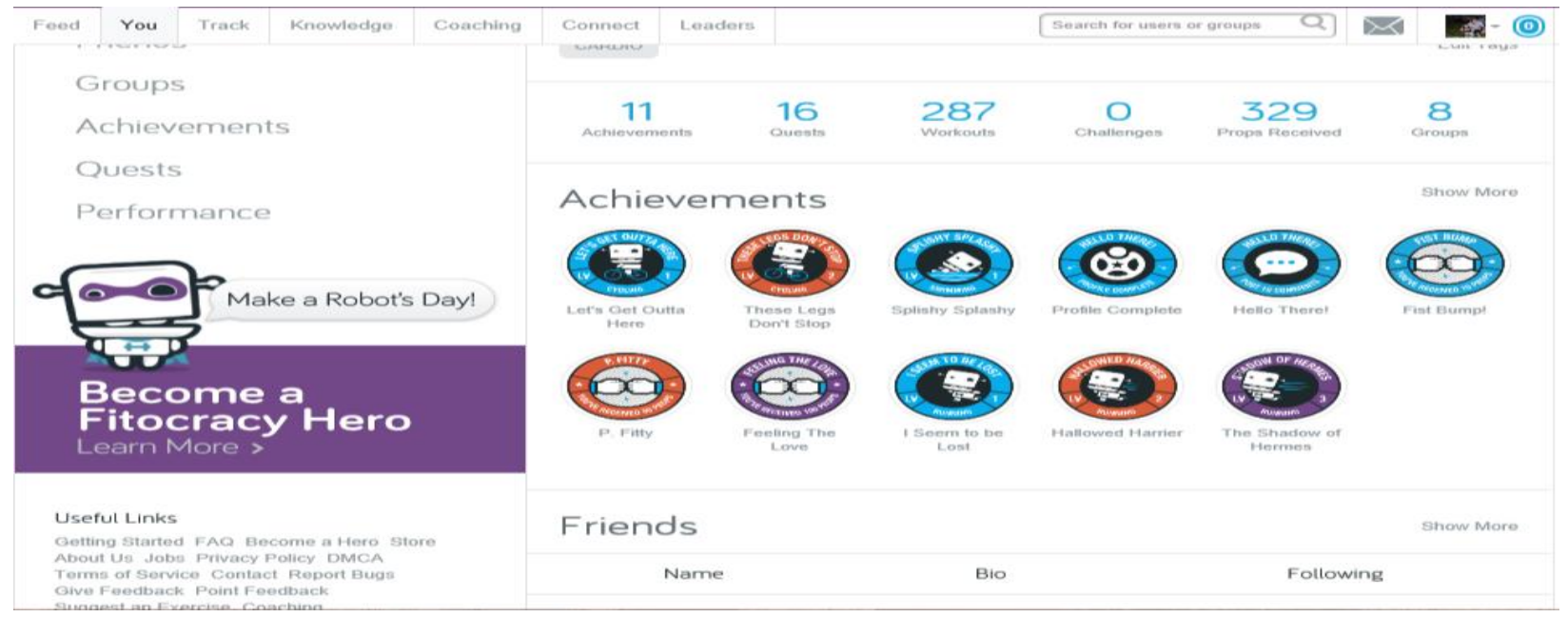

Figure 5. This screenshot is an example of informational social support. 


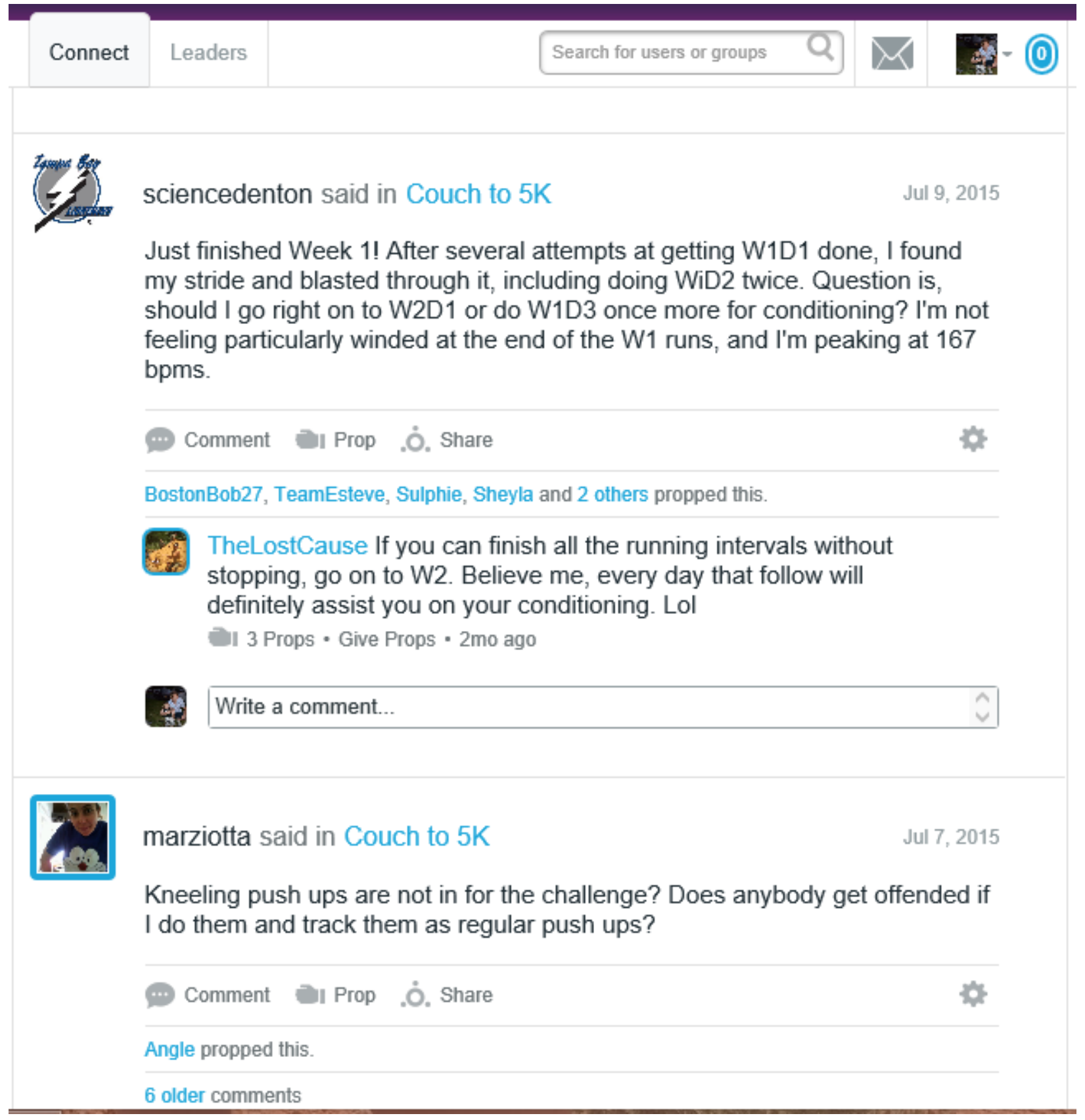




\section{References}

Ames, D. R., Rose, P., \& Anderson, C. P. (2006). The NPI-16 as a short measure of narcissism. Journal of Research in Personality, 40, 440-450.

Annesi, J. (2016, Jun 6). Weight loss success: How exercise helps control eating. On health. Retrieved from https://blogs.biomedcentral.com/on-health/2016/06/06/weight-losssuccess-how-exercise-helps-control-eating/

Atkin, D. (1993). Uses of cable TV amidst a multimedia environment. Telematics \& Informatics, 10, 51-60.

Atkin, D., Hunt, D., \& Lin, C. A. (2015). Diffusion theory in the new media environment: Towards an integrated model of technology adoption. Mass Communication \& Society, $18,623-650$.

Augemberg, K. (2012). Building that perfect quantified self app: Notes to developers, Part 1. Retrieved from www.measuredme.com/2012/10/building-that-perfectquantified-self-app

Ayabe, M., Brubaker, P. H., Mori, Y., Kumahara, H., Kiyonaga, A., Tanaka, H., \& Aoki, J. (2010). Self-monitoring moderate-vigorous physical activity versus steps/day is more effective in chronic disease exercise programs. Journal of Cardiopulmonary Rehabilitation and Prevention, 30(2), 111-115.

Bandura, A. (1986). Social foundations of thought and action: A social cognitive theory. Englewood Cliffs, NJ: Prentice-Hall.

Bandura, A. (1997). Self-efficacy: The exercise of control. New York, NY: Freeman.

Bann, C., Kobau, R., Lewis, M., Zack, M., Luncheon, C., \& Thompson, W. (2012). Development and psychometric evaluation of the public health surveillance well-being scale. Quality of Life Research, 21(6), 1031-1043. 
Bartsch, A., Mangold, R., Viehoff, R., \& Vorderer, P. (2006). Emotional gratifications during media use - An integrative approach. Communications: The European Journal of Communication Research, 31(3), 261-278.

Benisovich, S. V., Rossi, J. S., Norman, G J., \& Nigg, C. R. (1998). A multidimensional approach to exercise self-efficacy: Relationship with exercise behavior and attitudes towards exercise. Paper presented at the Annual Meeting of the New England Psychological Association, Boston, MA.

Boulianne, S. (2015). Social media use and participation: A meta-analysis of current research. Information, Communication \& Society, 18(5), 524-538.

Breton, E.R., Fuemmeler, B.F., \& Abroms, L.C. (2011). Weight loss-there is an app for that! But Does it adhere to evidence informed practices? Translational Behavioral Medicine, 1(4), 533-529.

Buffardi, L., \& Campbell, W. (2008). Narcissism and social networking web sites. Personality and Social Psychology Bulletin, 34(10), 1303-1314.

Carels, R. A., Darby, L. A., Rydin, S., Douglass, O. M., Caccaiapaglia, H. M., \& O’Brien, W. H. (2005). The relationship between self-monitoring, outcome expectancies, difficulties with eating and exercise, and physical activity and weight loss treatment outcomes. Annals of Behavioral Medicine, 30(3) 182-190.

Carr, L. J., Dunsiger, S. I., Lewis, B., Ciccolo, J. T., Hartman, S., Bock, B., ... \& Marcus, B. H. (2013). Randomized controlled trial testing an internet physical activity intervention for sedentary adults. Health Psychology, 32(3), 328. 
Charney, T., \& Greenberg, B. S. (2002). Uses and gratifications of the Internet. In C. Lin \& D. Atkin (Eds.), Communication technology and society: Audience adoption and uses (pp. 379-407). New York, NY: Hampton Press.

Choi, A., \& Chen, W. (2006, June). Online social support and intercultural adaptation. Paper presented at 54th Annual Meeting of the International Communication Association, Dresden, Germany (pp. 19-23).

Cowan, L. T., Van Wagenen, S. A., Brown, B. A., Hedin, R. J., Seino-Stephan, Y., Hall, P. C., \& West, J. H. (2013). Apps of steel: Are exercise apps providing consumers with realistic expectations? A content analysis of exercise apps for presence of behavior change theory. Health Education \& Behavior, 40(2), 133-139.

Dam, L., Roy, D., Atkin, D., \& Rodgers, D. (2018). Applying a uses and gratifications approach to health app adoption and use. Journal of Broadcasting \& Electronic Media, 62, 654672.

Eastin, M. S., \& LaRose, R. (2000). Internet self-efficacy and the psychology of the digital divide. Journal of Computer-Mediated Communication, 6(1), JCMC611.

Embacher Martin, K., McGloin, R., \& Atkin, D. (2018). Body dissatisfaction, neuroticism, and female sex as predictors of calorie-tracking app use amongst college students. Journal of American College Health, 66(7), 608-616.

Fogg, J. (2003). Persuasive technology: Using computers to change what we think and do. San Francisco, CA: Morgan Kaufman Publishers.

Helsel, D. L., Jakicic, J. M., \& Otto, A. D. (2007). Comparison of techniques for selfmonitoring, eating and exercise behaviors on weight loss in a correspondencebased intervention. Journal of the American Dietetic Association, 107(10), 1807-1810. 
Holbert, R. L., \& Stephenson, M. T. (2003). The importance of indirect effects in media effects research: Testing for mediation in structural equation modeling. Journal of Broadcasting \& Electronic Media, 47(4), 556-572.

Hunt, D. S., Lin, C. A., \& Atkin, D. J. (2014). Photo-messaging: Adopter attributes, technology factors and use motives. Computers in Human Behavior, 40, 171-179.

Ingledew, D. K., \& Markland, D. (2008). The role of motives in exercise participation. Psychology and Health, 23, 807-828.

Katz, E., Blumler, J., \& Gurevitch, M. (1974). Utilization of mass communication by the individual. In J. Blumler \& E. Katz (Eds.), The uses of mass communication: Current perspectives on gratifications research (pp. 19-34). Beverly Hills, CA: Sage.

Kim, C. K., Jun, M., Han, J., \& Kim, J. Y. (2013). Antecedents and outcomes of attachment towards smartphone applications. International Journal of Mobile Communications, 11, 393-411.

Krebs, P., \& Duncan, D. T. (2015). Health app use among US mobile phone owners: A national survey. JMIR mHealth and uHealth, 3(4), e101.

Krishnan, A., \& Atkin, D. (2014). Individual differences in social networking site users: The interplay between antecedent variables and the subsequent effect on level of activity. Computers in Human Behavior, 40, 111-118.

LaChausse, R. G. (2006). Motives of competitive and non-competitive cyclists. Journal of Sport Behavior, 29(4), 304-314.

Lagoe, C., \& Atkin, D. (2015). Health anxiety in the digital age: An exploration of psychological determinants of online health information seeking. Computers in Human Behavior, 52, 484-491. 
Leung, L., \& Wei, R. (1998). Factors influencing the adoption of interactive TV in Hong Kong: Implications for advertising. Asian Journal of Communication, 8(2), 124-147.

Lin, C. A. (2009). Exploring the online radio adoption decision-making process: Cognition, attitude, and technological fluidity. Journalism \& Mass Communication Quarterly, 86(4), 884-899.

Lin, C., Atkin, D., Cappotto, C., Davis, C., Dean, J., Eisenbaum, J., ...Vidican, S. (2015). Ethnicity, the digital divide and uses of the Internet for health information. Computers in Human Behavior, 51, 216-223.

Markland, D., \& Ingledew, D. K. (1997). The measurement of exercise motives: Factorial validity and invariance across gender of a revised Exercise Motivations Inventory. British Journal of Health Psychology, 2, 361-376.

Masters, K. S., \& Ogles, B. M. (1995). An investigation of the different motivations of marathon runners with varying degrees of experience. Journal of Sport Behavior, 18, 69-80.

McGloin, R., Embacher, K., \& Atkin, D. (2017). Health and exercise-related predictors of distance-tracking app usage. Health Behavior and Policy Review, 4(4), 306-317.

Netimperative. (2017). Health and fitness app usage "grew 330\% in just 3 years." Retrieved Feb. 28, 2018, from http://www.netimperative.com/2017/09/health-fitness-app-usage-grew330-just-3-years/

Mullinix, K. J., Leeper, T. J., Druckman, J. N., \& Freese, J. (2015). The generalizability of survey experiments. Journal of Experimental Political Science, 2(2), 109-138.

Palmgreen, P., Wenner, L. A., \& Rosengren, K. E. (1985). Uses and gratifications research: The past ten years. In K. E. Rosengren, L. A. Wenner, \& P. Palmgreen (Eds.), Media gratifications research: Current perspectives (pp. 11-37). Beverly Hills, CA: Sage. 
Patel, M. S., Asch, D. A., \& Volpp, K. G. (2015). Wearable devices as facilitators, not drivers, of health behavior change. JAMA, 313(5), 459-460.

Rabin, C., \& Bock, B. (2011). Desired features of smartphone applications promoting physical activity. Telemedicine and e-Health, 17(10), 301-303.

Raskin, R., \& Terry, H. (1988). A principal-components analysis of the Narcissistic Personality Inventory and further evidence of its construct validity. Journal of Personality \& Social Psychology, 54(5), 890-902.

Rogers, E. (2003). Diffusion of Innovations. (5th Ed.). New York, NY: The Free Press.

Rosengren, K. E. (1974). Uses and gratifications: A paradigm outlined. In J. G. Blumler \& E. Katz (Eds.), The uses of mass communications: Current perspectives on gratifications research (pp. 269-286). Beverly Hills, CA: Sage.

Rubin, A. M. (2009). Uses and gratifications perspective on media effects. In J. Bryant \& M. B. Oliver (Eds.), Media effects: Advances in theory and research (pp. 165-184). New York, NY: Routledge.

Sherry, J., \& Lucas, K. (2003). Video game uses and gratifications as predictors of use of game preference. Paper presented at the annual meeting of the International Communication Association, San Diego, CA.

Strizhakova, Y., Kang, Y., \& Buck, R. (2007). Assessment of emotional gratifications: Development of the EGRATS Scale. Media Psychology, 10(1), 91-112.

Sundar, S. S., \& Limperos, A. M. (2013). Uses and grats 2.0: New gratifications for new media. Journal of Broadcasting \& Electronic Media, 57(4), 504-525.

Walther, J., \& Boyd, S. (2002). Attraction to computer-mediated social support. In C. Lin (Ed). Communication technology ad society: New media adoption 
and uses (pp. 152-188). Cresskill, NJ: Hampton.

Wei, R. (2013). Mobile media: Coming of age with a big splash. Mobile media \& Communication, 1 (1), 50-56.

Wei, R., Karlis, J., \& Haught, M. J. (2012, May). Apps, apps, and more apps: A uses and gratification study of app use. -Paper presented at the International Communication Association, Phoenix, AZ.

Weiss, A. (2013). Exploring news apps and location-based services on the smartphone. Journalism \& Mass Communication Quarterly, 90(3), 435-456.

Zillmann, D. (2000). Mood management in the context of selective exposure theory. In M. F. Roloff (Ed.), Communication Yearbook, 23 (pp. 103-123). Thousand Oaks, CA: Sage. 